\title{
A multiscale method for optimising surface topography in elastohydrodynamic lubrication (EHL) using metamodels
}

\author{
G. N. de Boer ${ }^{1}$ - L. Gao ${ }^{1}$ - R. W. Hewson ${ }^{1}$ - H. M. Thompson ${ }^{2}$. \\ N. Raske $^{3} \cdot$ V. V. Toropov ${ }^{3}$
}

Received: 1 October 2015 /Revised: 11 December 2015 / Accepted: 22 December 2015 /Published online: 2 April 2016

(C) The Author(s) 2016. This article is published with open access at Springerlink.com

\begin{abstract}
The frictional performance of a bearing is of significant interest in any mechanical system where there are lubricated surfaces under load and in relative motion. Surface topography plays a major role in determining the coefficient of friction for the bearing because the size of the fluid film and topography are of a comparable order. The problem of optimising topography for such a system is complicated by the separation in scales between the size of the lubricated domain and that of the topography, which is of at least one order of magnitude or more smaller. This paper introduces a multiscale method for optimising the small scale topography for improved frictional performance of the large scale bearing. The approach fully couples the elastohydrodynamic lubrication at both scales between pressure generated in the lubricant and deformation of the bounding surfaces. Homogenised small scale data is used to inform the large scale model and is represented using Moving Least Squares metamodels calibrated by cross validation. An optimal topography for a minimum coefficient of friction for the bearing is identified and comparisons made of local minima in the response,
\end{abstract}

G. N. de Boer

g.de-boer@imperial.ac.uk

1 Department of Aeronautics, Imperial College London, London SW7 2AZ, UK

2 School of Mechanical Engineering, University of Leeds, Leeds LS2 9JT, UK

3 School of Engineering and Materials Science, Queen Mary University of London, London E1 4NS, UK where very different topographies with similar frictional performance are observed. Comparisons of the optimal topography with the smooth surface model demonstrated the complexity of capturing the non-linear effect of topography and the necessity of the multiscale method in capturing this. Deviations from the smooth surface model were quantified by the metamodel coefficients and showed how topographies with a similar frictional performance have very different characteristics.

Keywords Multiscale method - Bracketing optimisation · Surface topography $\cdot$ Metamodelling $\cdot$ Moving Least Squares · Cross Validation
Abbreviations
BVP Boundary Value Problem
CFD Computational Fluid Dynamics
CV Cross Validation
DOE Design of Experiments
EHL Elastohydrodynamic Lubrication
FSI Fluid structure Interaction
GA Genetic Algorithm
HMM Heterogeneous Multiscale Methods
IVP Initial Value Problem
k-CV k-fold Cross Validation
LOO-CV Leave-One-Out Cross Validation
MLS Moving Least Squares
OLHC Optimum Latin Hypercube
PTFE Polytetrafluoroethlyene
RMSE Root Mean Squared Error
Nomenclature
A MLS matrix
b Right-hand-side variable in the MLS operation 


\begin{tabular}{|c|c|}
\hline $\mathrm{C}_{1-8}$ & MLS coefficients \\
\hline $\mathrm{E}$ & Young's modulus \\
\hline $\mathrm{E}^{\prime}$ & Equivalent Young's modulus \\
\hline $\mathrm{h}$ & Undeformed film thickness \\
\hline $\mathrm{g}$ & Film gap \\
\hline G & Normalised film gap \\
\hline I & Identity matrix \\
\hline $\mathrm{k}$ & Fold size \\
\hline $\mathrm{k}_{1}$ & Local stiffness \\
\hline $\mathbf{K}$ & Stiffness matrix \\
\hline $\mathrm{L}$ & Cell length \\
\hline $\mathrm{L}_{\mathrm{p}}$ & Pad length \\
\hline $\mathrm{N}$ & Size of DOE \\
\hline $\mathrm{p}$ & Pressure \\
\hline $\mathrm{p}^{*}$ & Load per unit area \\
\hline $\mathrm{p}_{\mathrm{s}}$ & Small scale pressure \\
\hline $\mathrm{P}$ & Normalised pressure \\
\hline$\frac{d p}{d x}$ & Pressure gradient \\
\hline$\Delta \mathrm{p}$ & Pressure jump \\
\hline$q$ & Mass flow rate per unit depth \\
\hline $\mathrm{Q}$ & Normalised mass flow rate per unit depth \\
\hline $\mathrm{r}$ & Normalised Euclidean distance \\
\hline s & Small scale film thickness \\
\hline$\Delta \mathrm{s}$ & Deformation of the small scale film thickness \\
\hline $\mathrm{t}$ & Pad thickness \\
\hline$t^{\prime}$ & Equivalent thickness \\
\hline $\mathrm{u}_{\mathrm{s}} \mathrm{v}_{\mathrm{s}}, \mathrm{w}_{\mathrm{s}}$ & Small scale velocity components \\
\hline $\mathrm{U}$ & Moving wall velocity \\
\hline $\mathrm{w}$ & Moving least squares weight \\
\hline $\mathrm{W}$ & Load capacity \\
\hline $\mathrm{W}_{\text {rep }}$ & Required load capacity \\
\hline $\mathrm{x}$ & Large scale coordinate direction \\
\hline $\mathrm{x}_{\mathrm{s}}, \mathrm{y}_{\mathrm{s}}, \mathrm{z}_{\mathrm{s}}$ & Small scale coordinate directions \\
\hline$\alpha$ & Topography amplitude \\
\hline$\gamma$ & $\begin{array}{l}\text { Left-hand-side variable in the MLS } \\
\text { operation }\end{array}$ \\
\hline$\Gamma$ & Vector of MLS multipliers \\
\hline$\delta$ & Deformation \\
\hline$\delta_{\mathrm{t}}$ & Topography \\
\hline$\eta_{0}$ & Ambient viscosity \\
\hline$\eta_{\mathrm{s}}$ & Small scale viscosity \\
\hline$\theta$ & Closeness of fit parameter \\
\hline$\mu$ & Coefficient of friction \\
\hline$v$ & Poisson's ratio \\
\hline$\rho_{0}$ & Ambient density \\
\hline$\rho_{\mathrm{s}}$ & Small scale density \\
\hline$\tau$ & Shear stress \\
\hline$\varphi$ & Tilt angle \\
\hline$\psi$ & Small scale topography parameter \\
\hline a & Inlet \\
\hline & Outlet \\
\hline & Assessment location \\
\hline
\end{tabular}

\section{Introduction}

Elastohydrodynamic Lubrication (EHL) describes the formation of a lubricated film between two machine elements which are under load and in relative motion to each other. The pressurisation of the lubricant in the EHL regime is large enough to cause deformation of the mating surfaces, which in turn leads to a change in the lubricating film thickness. Fluid film lubrication is conventionally modelled using the Reynolds equation (Cameron 1971) based on the lubrication between two nearly parallel smooth surfaces. EHL exists in mechanical systems such as gear teeth, bearings, rubber seals and car tyres on a wet road (Dowson 1999). One of the design challenges for such systems is to control the coefficient of friction between the mating surfaces, typically to reduce it for machine components and increase it where traction is required. Tzeng and Saibel (1967), Patir and Cheng (1978), Etsion (2005), de Kraker et al. (2007), Sahlin et al. (2010), de Boer et al. (2014) have found that texturing bearing surfaces can have a significant effect on the coefficient of friction of lubricated contacts compared to the smooth bearing case. Despite the significant work showing potential performance improvements in terms of friction there is still a need for an efficient computational approach to analyse bearing topography and more importantly to use such analysis for design optimisation.

Minimising friction in a textured EHL contact is complicated by the fact that it is a multi-scale problem where the scale of the overall lubricated domain is typically an order of magnitude or more greater than that of the topographical features (Gohar 2001). This disparity of scales means that it is infeasible to computationally resolve the small scale features as well as the large scale bearing domain. This is especially true when (i) the assumptions on which the Reynolds equation is based (Cameron 1971) break down due to the local topography and the bearing surfaces no longer being nearly parallel, (ii) the deformation of the bearing geometry under the influence of pressurised lubricant is also considered as is the case in EHL.

To resolve this challenging problem, multiscale modelling techniques have been developed to address the computational requirements in obtaining numerical solutions to the problem. A range of multiscale approaches have been proposed to solve this problem, including the Flow Factors approach (Patir and Cheng 1978) and that based on the Heterogenous Multiscale Method (Gao and Hewson 2012). These approaches share significant similarities, whereby the small scale flow around a single (or single set of) periodic topographical feature(s) is resolved for a range of potential large scale conditions, the homogenised solution of which is then used to obtain the large scale solution. The solution of the small scale problem has been based on lubrication (Sahlin et al. 2010), Stokes (de 
Kraker et al. 2007) and Navier-Stokes flow models (de Boer et al. 2014; Gao et al. 2015). A similar mulitscale approach has also been used to model coating flows where topographical features are present (Hewson et al. 2011).

Data communication between the length-scales of a multi-scale method can be achieved in several ways. Serial coupling methods pass parameters between the scales and determine the effective macroscopic parameters from the micro-scale model in a pre-processing step and use the macroscopic model in the computations, see de Kraker et al. (2007) in the context of EHL modelling. In concurrent coupling methods, however, the micro-scale and macro-scale models are linked dynamically during the overall computation (Abraham et al. 1999) and it is therefore important to build a fast input/output relationship between models at the different scales (Roux et al. 1998). The use of metamodels to represent the small-scale data is becoming increasingly popular. Benke et al. (2009), for example, used metamodels for the atomic structure of DNA molecules to determine the parameters of the mechanical structures within a multiscale model of polymer migration in DNA-laden flows, and Writz et al. (2015) recently proposed the use of kernel methods to provide fast metamodels for multiscale modelling of the human spine. The general metamodelling approach is divided into two stages: a computationally expensive offline stage where the metamodelling parameters are calibrated against a set of training data; and an online computing stage comprising fast execution of the resulting metamodel within the multi-scale modelling framework.

de Boer et al. (2014) used multi-dimensional metamodels for representing efficiently the small scale model within a macroscopic EHL model. Their metamodel approximations were based on the Moving Least Squares (MLS) method and were calibrated using Cross Validation (CV) techniques combined with efficient space-filling Design of Experiments (DOE) (Loweth et al. 2011). The present study will exploit the synergies between multiscale EHL analysis and response surface enabled optimisation in order to demonstrate a means of achieving multiscale design optimisation across the scales. This will be demonstrated by the optimisation of small scale bearing topography for a minimal coefficient of friction of the bearing system.

The paper is organised as follows. The multiscale EHL method and metamodelling strategies are introduced in Section 2. Numerical methods are described in Section 3. In Section 4 the validation and performance of the metamodel is demonstrated and the optimal substrate topography leading, to minimal frictional drag, identified. Conclusions are drawn in Section 5.

\section{Theory}

\subsection{Problem formulation}

The optimisation problem described here represents one frequently encountered in the field of tribology, that of how to reduce the coefficient of friction in an EHL contact for a constant applied load. The method described is based on the Heterogeneous Multiscale Methods (E et al. 2007) whereby homogenised information obtained from periodic small scale models (topography) is used to derive a relationship for the large scale model (bearing). An overview of the two-scale method for EHL is given here, a full description can be found in de Boer et al. (2014).

A simple large scale geometry will be considered, that of a 2D linear slider bearing as shown below in Fig. 1. The domain between inlet and outlet is fully flooded with lubricant, the lower wall of the bearing moves in the $\mathrm{x}$ coordinate direction with velocity $U$ and does not deform, the upper surface is stationary and deformable.

Here the large scale geometry, specifically the pad length $\mathrm{L}_{\mathrm{p}}$ and tilt angle $\varphi$ are kept constant, however they could be varied in order to maximise bearing performance, as would be undertaken for single scale optimisation. To demonstrate the means by which optimisation is undertaken across scales a small scale geometry parameter $\psi$ is defined. The multiscale optimisation problem is subsequently given by (1)-(3):

$\min _{\psi}(\mu)$

$0 \leq \psi \leq 1$

$\mathrm{W}\left(\mathrm{h}_{\mathrm{b}}\right)-\mathrm{W}_{\text {req }}=0$

where the objective $\mu$ is the bearing coefficient of friction and the constraint $\mathrm{W}$ is the bearing load capacity, as calculated by (4) and (5) respectively. $W_{\text {req }}$ is the required bearing load which is achieved by varying the minimum undeformed film thickness for the bearing $h_{b}$ to give the undeformed film thickness $\mathrm{h}$ :

$$
\begin{aligned}
& \mu=\frac{1}{W} \int_{0}^{L_{p}} \tau\left(\frac{d p}{d x}, p, g, \psi\right) d x \\
& W=\int_{0}^{L_{p}} p^{*}\left(\frac{d p}{d x}, p, g, \psi\right) d x
\end{aligned}
$$

$\tau\left(\frac{d p}{d x}, p, g, \psi\right)$ and $p^{*}\left(\frac{d p}{d x}, p, g, \psi\right)$ are the large scale shear stress and load per unit area which are obtained from the small scale simulations. The variables $\frac{d p}{d x}, p$ and $g$ are the homogenised pressure gradient, pressure, and lubricated film gap respectively. These three variables along with the small scale geometry parameter $\psi$ define the small scale simulations as described in Section 2.2. 


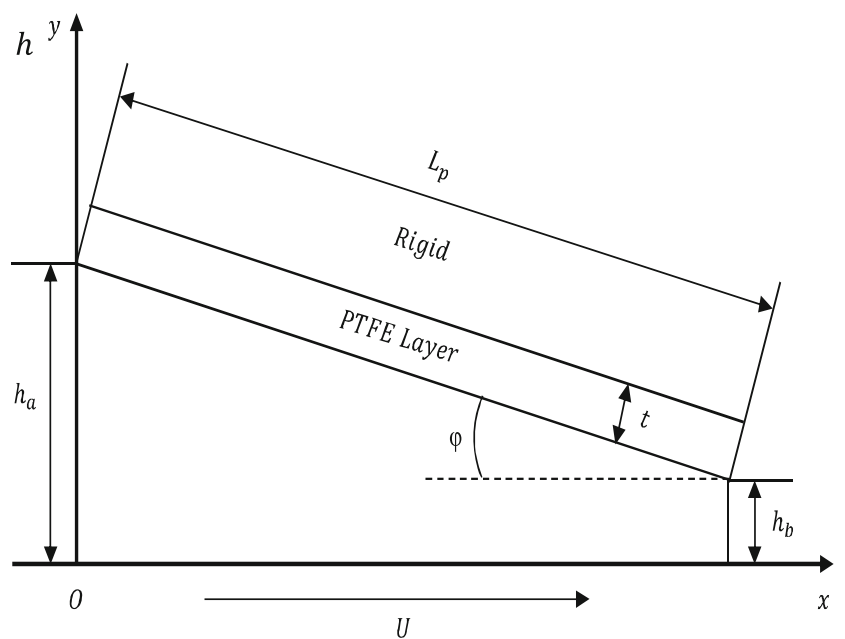

Fig. 1 Large scale bearing geometry

To solve the large scale problem and evaluate (i) the load per unit area (which differs slightly from the large scale pressure due to the homogenisation approach) and (ii) shear stress, the large scale governing equations for the pressure (6) and lubricant flow rate (7) are solved:

$\frac{\mathrm{dp}}{\mathrm{dx}}=\frac{\mathrm{dp}}{\mathrm{dx}}(\mathrm{p}, \mathrm{g}, \mathrm{q}, \psi)$

$\frac{\mathrm{dq}}{\mathrm{dx}}=0$

The equation for the pressure gradient is also obtained from the small scale simulations, where $\mathrm{q}$ is the mass flow rate per unit depth. The large scale solution to (6) and (7) is obtained with Dirichlet boundary conditions for zero (ambient) pressure (8) at both inlet and outlet:

$\mathrm{p}_{\mathrm{a}}=\mathrm{p}_{\mathrm{b}}=0$

The bearing deformation is accounted for at both the large and small scale in order to account for non-local deformation (deformation at a surface location due to a pressure at a different location on the pad surface) and the micro-elastohydrodynamic surface deformation (deformation of the small scale domain due to small scale variations in the pressure profile). It is based on the elastic deformation $\delta$ which would be encountered in the smooth case and is found via a matrix operation, where the influence of pressure on displacement decreases with the distance from the point at which it is applied. The total deformation influence matrix $\mathbf{K}$, or deformation coefficient matrix, is calculated using elasticity theory based on the thickness of the Polytetrafluoroethylene (PTFE) pad t (Rodkiewicz and Yang 1995). The relationship describing how load per unit area $\mathrm{p}^{*}$ relates to deformation is given by $\delta=\mathbf{K}$ * .
This can be rewritten such that total deformation is the sum of two separate terms as given by (9):

$\delta=\left(\mathbf{K}-\mathrm{k}_{1} \mathbf{I}\right) \mathrm{p}^{*}+\mathrm{k}_{1} \mathbf{I} \mathrm{p}^{*}$

In (9), $k_{1}$ is the local stiffness which is subsequently modelled at the small scale, see Section 2.2. The term $k_{1} I p$ accounts for local deformation (deformation due to pressure at that location) and $\left(\mathbf{K}-\mathrm{k}_{1} \mathbf{I}\right)$ p* the remaining deformations (deformation due to pressure at all other locations). The film gap (g) becomes the sum of the undeformed film thickness $h$ and non-local deformation allowing local deformation to be considered at the small scale (including micro-EHL where deformation of individual topographical features may occur). (6), (7) and (10) are coupled and solved iteratively until convergence in the pressure distribution is reached:

$\mathrm{g}=\mathrm{h}+\left(\mathbf{K}-\mathrm{k}_{1} \mathbf{I}\right) \mathrm{p}^{*}$

In defining the optimisation problem the variable defining the small scale geometry $\psi$ is included in the functional terms defining the pressure gradient $\frac{\mathrm{dp}}{\mathrm{dx}}(\mathrm{p}, \mathrm{g}, \mathrm{q}, \psi)$, shear stress $\tau$ $\left(\frac{d p}{d x}, p, g, \psi\right)$ and load per unit area $p^{*}\left(\frac{d p}{d x}, p, g, \psi\right)$. This is an important distinction when compared with the analysis case as it means that a representation is required of how the small scale behaves with changes in both the local operating conditions $\frac{\mathrm{dp}}{\mathrm{dx}}, \mathrm{p}, \mathrm{g}, \mathrm{q}$ as well as the optimisation problem's design variables $\psi$ (in this case). This representation of the small scale problem is achieved using a metamodel of the small scale behaviour and for this case is therefore 4dimensional.

\subsection{Small scale simulations}

A series of small scale simulations were undertaken on which the metamodel was constructed. These small scale simulations were a set of Fluid-Structure Interaction (FSI) problems with the lubricant flow modelled using the Navier-Stokes equations and the local deformation and micro-EHL simulated by an equivalent column of elastic bearing material.

The small scale simulations use an equivalent thickness $t^{\prime}$ of the solid domain to ensure that the resulting deformation due to fluid pressure is equal to the column deformation achieved from the local stiffness required $\mathrm{k}_{1}$ at the large scale under the same pressure (11). The equivalent elastic modulus $\mathrm{E}^{\prime}$ is derived to represent the mechanical properties of the large scale stiffness to a fully constrained column of bearing material in three-dimensions at the small scale (12):

$\mathrm{t}^{\prime}=\mathrm{k}_{1} \mathrm{E}^{\prime}$

$\mathrm{E}^{\prime}=\frac{(1-\vartheta) \mathrm{E}}{(1+v)(1-2 v)}$ 
where $\mathrm{E}$ and $v$ are the Young's modulus and Poisson's ratio of the bearing material (PTFE) respectively. By applying an equivalent thickness to the problem the small scale FSI is accurately described whilst maintaining the required stiffness properties at the large scale and of the bearing surface. Structural mechanics at the small scale are considered by implementation of a 3D model for a linearly elastic solid, the material is assumed homogenous and isotropic. The upper boundary of the spring column is fully constrained. The sides of the spring column are constrained normal to the surface. The fluid/solid interface is loaded by pressure generated from the fluid flow simulations.

The small scale fluid geometry is defined by a cell length $\mathrm{L}$ in both $\mathrm{x}_{\mathrm{s}}$ and $\mathrm{y}_{\mathrm{s}}$ coordinate directions, this length scale must be an order of magnitude or more smaller than the pad length $\mathrm{L}_{\mathrm{p}}$. The $\mathrm{z}_{\mathrm{s}}$ coordinate is aligned with the film gap $\mathrm{g}$. Topography $\delta_{t}$ is defined by the small scale geometry parameter $\psi$ and the topography amplitude $\alpha(13)$, this is summed with $\mathrm{g}$ to form the small scale film thickness $\mathrm{s}$. The parameter $\psi$ controls the ratio of longitudinal to transverse waviness in the topography response, at $\psi=0$ topography is longitudinal and at $\psi=1$ topography is transverse to the direction of motion of the bearing surface:

$$
\begin{aligned}
& \delta_{\mathrm{t}}=\frac{\alpha}{2}\left[\psi\left(\sin \left(2 \pi \frac{\mathrm{x}_{\mathrm{s}}}{\mathrm{L}}\right)+1\right)\right. \\
& \left.+(1-\psi)\left(\sin \left(2 \pi \frac{\mathrm{y}_{\mathrm{s}}}{\mathrm{L}}\right)+1\right)\right]
\end{aligned}
$$

A diagram showing the small scale geometry with both solid and fluid domains is shown in Fig. 2.

The small scale flow is modelled using Computational Fluid Dynamics (CFD) where the flow is considered steady, laminar, compressible and isothermal as described by the Navier-Stokes

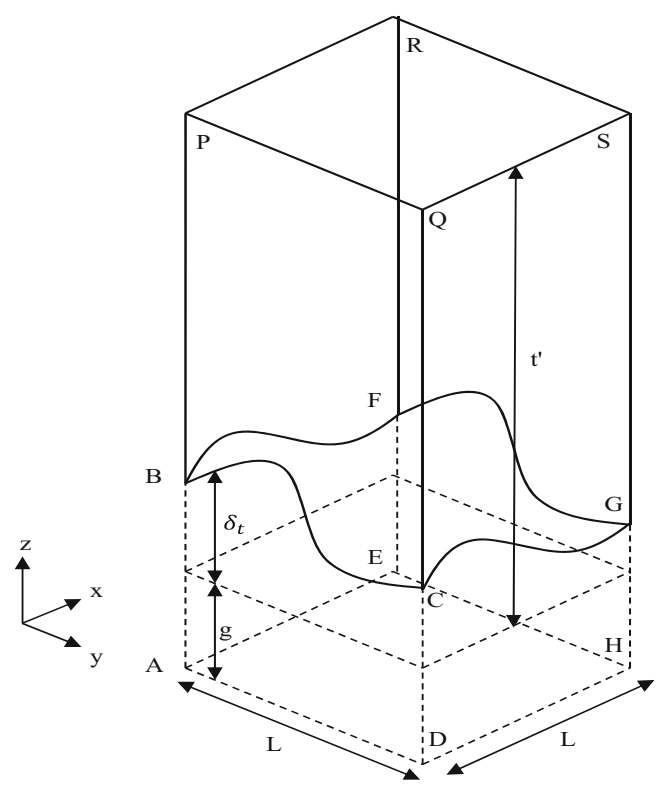

Fig. 2 Small scale fluid (dashed) and solid (full) equations. The lubricant density $\rho_{\mathrm{s}}$ varies barotropically as described by the Dowson-Higginson equation (Dowson and Higginson 1966). Piezoviscosity is modelled using the Roelands equation (Roelands 1966) and non-Newtonian (shear-thinning) behaviour is considered using the Ree-Eyring model to give the lubricant viscosity $\eta_{\mathrm{s}}$ (Johnson and Tevaarwerk 1977). The interface between the fluid and solid is a no-slip wall, the lower surface is a moving wall with velocity $\mathrm{U}$, the remaining boundaries are subject to the near-periodic boundary condition. The pressure $\mathrm{p}_{\mathrm{s}}$ on each set of nearperiodic boundaries experiences a jump $\Delta \mathrm{p}$ over the domain. Velocity components $\mathrm{u}_{\mathrm{s}}, \mathrm{v}_{\mathrm{s}}, \mathrm{w}_{\mathrm{s}}$ are scaled at the boundaries to account for the difference in density (due to pressure at the boundaries) and area (due to deformation of the boundaries).

The generation of the data points from which to generate the response surfaces to be used in the optimisation of the tribology problem requires the homogenisation of the small scale geometry, it is this homogenisation which leads to the requirement that the small scale domain is at least an order of magnitude smaller than that of the large scale problem, $\mathrm{L} \ll \mathrm{L}_{\mathrm{p}}$. This is analogous to the separation of scales between the bearing length and lubricated gap in derivation of the Reynolds equation (the accurate description of smooth surface lubrication) which states that the film thickness varies gradually along the length of the bearing.

Given a pressure constraint, initial gap, and small scale geometry parameter, the solution fields for pressure and velocity can be obtained by solving for the small scale model. Coupling of structural mechanics and CFD is achieved through an Arbitrary Lagrangian-Eulerian approach in a Finite Element (FE) simulation. The homogenised pressure gradient $\frac{\mathrm{dp}}{\mathrm{dx}}$ over a unit cell is calculated using (14), and the mass flow rate per unit depth $\mathrm{q}$ is determined from (15) on the periodic boundary normal to the sliding direction:

$\frac{\mathrm{dp}}{\mathrm{dx}}=\frac{\Delta \mathrm{p}}{\mathrm{L}}$

$q=\frac{1}{L} \int_{0}^{s+\Delta s} \int_{0}^{L} \rho_{s} u_{s} d y_{s} d z_{s}$

Pressure is not uniformly distributed in the small scale domain and so an average cell pressure $\mathrm{p}^{*}$ is derived which describes the load per unit area and from which deformation $\delta$ and load capacity $\mathrm{W}$ are determined at the large scale (16). A similar expression exists for the shear stress $\tau$ from which the coefficient of friction $\mu$ is found (17). Both (16) and (17) are calculated on the sliding wall boundary:

$$
\begin{aligned}
& \mathrm{p}^{*}=\frac{1}{\mathrm{~L}^{2}} \int_{0}^{\mathrm{L}} \int_{0}^{\mathrm{L}} \mathrm{p}_{\mathrm{s}} \mathrm{dx}_{\mathrm{s}} d \mathrm{y}_{\mathrm{s}} \\
& \tau=\frac{1}{\mathrm{~L}^{2}} \int_{0}^{\mathrm{L}} \int_{0}^{\mathrm{L}} \eta_{\mathrm{s}} \frac{\mathrm{du}_{\mathrm{s}}}{\mathrm{dz}_{\mathrm{s}}} \mathrm{dx}_{\mathrm{s}} d \mathrm{y}_{\mathrm{s}}
\end{aligned}
$$

where $\Delta \mathrm{s}$ is the deformation of the small scale film thickness. 


\subsection{Metamodel construction}

The construction of an accurate metamodel representing the terms $\frac{d p}{d x}(p, g, q, \psi), \tau\left(\frac{d p}{d x}, p, g, \psi\right)$ and $p^{*}\left(\frac{d p}{d x}, p, g, \psi\right)$ is critical to both the accurate analysis of the bearing performance and to permit the optimisation of the system. The first stage in constructing the metamodel is the generation of the simulation conditions (both design and operating variables). Creating a model of this nature requires a DOE which ensures the most efficient spread of simulations in the design space, as to reduce the number of simulations while allowing the response to be accurately described. An Optimal Latin Hypercube (OLHC) is used here to explore as much of the design space with as few designs as possible. This is generated using a permutation Genetic Algorithm (GA) to optimise the distance between all DOE points (Bates et al. 2004). This satisfies the AudzeEglais condition (Audze and Eglais 1977) which requires that the sum of the reciprocal of the squared distances between each DOE point and all others is a minimum.

MLS is derived from conventional weighted least squares model building where the weights do not remain constant but are functions of the normalised Euclidian distances from sample points to the point where the metamodel is evaluated (Choi et al. 2001). Coefficients in the basis function of the MLS approximation become functions of the design space and therefore describe how the metamodel varies from the least squares approximation (Breitkopf et al. 2005).

Reynolds equation (Cameron 1971) is used as the MLS basis function (18), when the coefficients $\mathrm{C}_{1-3}$ are unity the metamodel produces the result obtained directly from smooth surface lubrication (i.e. the pressure gradient Eq. (6) without topography and fluid flow phenomena). Expressions for the load per unit area (19) and shear stress (20) under smooth conditions are also obtained when $\mathrm{C}_{4-8}$ are unity. The MLS coefficients $\mathrm{C}_{1-8}$ therefore quantify the deviation from the smooth surface model when surface topography is included and are functions of the design space considered. In (18)-(20), $\rho_{0}$ and $\eta_{0}$ are the ambient density and viscosity of the lubricant respectively:

$$
\begin{aligned}
& \frac{d p}{d x}=\frac{12 \eta_{0}}{\rho_{0}\left(g+k_{1} p\right)^{3}}\left(\frac{C_{1} \rho_{0} U}{2}\left(g+k_{1} p\right)-C_{2} q\right)+C_{3}-1 \\
& p^{*}=C_{4} p+C_{5}-1 \\
& \tau=\frac{1}{g+k_{1} p}\left(\begin{array}{c}
\left.C_{6} U \eta_{0}+\frac{C_{7}}{2}\left(g+k_{1} p\right)^{2} \frac{d p}{d x}\right) \\
+C_{8}-1
\end{array}\right.
\end{aligned}
$$

A Gaussian decay function is defined for the MLS weights such that the influence of a known sample location will diminish exponentially with increasing distance to an assessment location, as is common in metamodel analyses (de
Boer et al. 2014; Loweth et al. 2011). Polynomial type decay functions can also be used to formulate the weights (Toropov et al. 2005). (26) describes how the weights corresponding to each know location $\mathrm{w}_{\mathrm{i}}$ are functions of the closeness of fit parameter $\theta$ and the normalised Euclidian distance to the assessment location $r_{i}$ :

$\mathrm{w}_{\mathrm{i}}=\exp \left(-\theta \mathrm{r}_{\mathrm{i}}^{2}\right) \quad \mathrm{i}=1, \ldots, \mathrm{N}$

where $\mathrm{N}$ is the total number of points in the sample set (DOE size) and $r_{i}$ is obtained from (22), in which capitals represented the normalised version of the corresponding lower case variables and tilde the assessment location of the metamodel:

$$
\begin{aligned}
\mathrm{r}_{\mathrm{i}}^{2}=\left(\tilde{\mathrm{P}}-\mathrm{P}_{\mathrm{i}}\right)^{2} & +\left(\tilde{\mathrm{G}}-\mathrm{G}_{\mathrm{i}}\right)^{2}+\left(\tilde{\mathrm{Q}}-\mathrm{Q}_{\mathrm{i}}\right)^{2} \\
& +\left(\tilde{\psi}-\psi_{\mathrm{i}}\right)^{2}
\end{aligned}
$$

By minimising the sum of squared error in the MLS approximation and known location values the coefficients in (18) can be determined at the assessment location by the matrix Eq. (23). Equation (23) is formulated by the series of Eqs. (24)-(27):

$$
\begin{aligned}
& \|\mathbf{A} \boldsymbol{\gamma}-\mathbf{b}\|=0 \\
& \mathrm{~b}_{\mathrm{i}}=\mathrm{w}_{\mathrm{i}}\left(\left(\frac{\mathrm{dp}}{\mathrm{dx}}\right)_{\mathrm{i}}+1\right) \\
& \mathrm{A}_{\mathrm{ij}}=\mathrm{w}_{\mathrm{i}} \Gamma_{\mathrm{ij}} \\
& \boldsymbol{\Gamma}_{\mathrm{i}}=\left[\begin{array}{lll}
\frac{6 \mathrm{U} \eta_{0}}{\left(\mathrm{~g}_{\mathrm{i}}+\mathrm{k}_{1} \mathrm{p}_{\mathrm{i}}\right)^{2}} & \frac{-12 \eta_{0} \mathrm{q}_{\mathrm{i}}}{\rho_{0}\left(\mathrm{~g}_{\mathrm{i}}+\mathrm{k}_{1} \mathrm{p}_{\mathrm{i}}\right)^{3}} & 1
\end{array}\right] \\
& \gamma_{\mathrm{j}}=\mathrm{C}_{\mathrm{j}} \quad \mathrm{j}=1, \ldots, 3
\end{aligned}
$$

Once the coefficients are calculated from (23) the MLS approximation can be determined by (18), substituting the coefficients and determining the polynomial expression for the assessment location. The same approach can be taken to assess the coefficients in (19) and (20).

The MLS approximation can be tuned to provide a more local or global response, this allows MLS to smooth numerical noise and provide an accurate approximation over the entire design space (Levin 1998). This is achieved by adjusting the closeness of fit parameter $\theta$. At $\theta=0$ the weights are all unity resulting in conventional least squares regression, the upper to limit to $\theta$ is infinity or until overfitting occurs (where no interpolation occurs between data points). The aim of tuning $\theta$ is to reduce the error in the known observations and that predicted by the metamodel, there exists an optimal $\theta$ which produces the lowest value of this error and to find this we must search through a range of $\theta$ until we observe a global minimum (Loweth et al. 2011).

To do this both the Leave-One-Out (LOO-) and k-fold (k-) CV methods are used (Loweth et al. 2011). For LOO-CV a range of $\theta$ is selected between 0 and an arbitrary upper bound 
which is large enough so ensure a global minimum is achieved. For every value of $\theta$ each observation is removed from the data set in turn and the approximation built from the remaining $\mathrm{N}-1$ points. The Root Mean Squared Error (RMSE) in the removed observations and those predicted at the removed locations are recorded over the total number of values. For k-CV instead of each point in turn being removed from the data set a random sample of size $\mathrm{k}$ is removed over many repeated steps, on each fold as the repeats are known the metamodel is built from the remaining $\mathrm{N}-\mathrm{k}$ points and the RMSE assessed in the metamodel predictions and known values at the removed locations. The average RMSE is then determined over the total number of folds, which is chosen to be large enough as to ensure no bias in the cross validation response toward certain regions of the design space. The values of $\theta$ which correspond to the minimum of the LOO$\mathrm{CV}$ and $\mathrm{k}-\mathrm{CV}$ errors are selected as the best fit. There is a compromise between the $\theta$ predicted by the two methods, both are at least capable of identifying the region near the optimal $\theta$ and so the choice made by the user on which value to use will not significantly affect the accuracy of the metamodel prediction.

The cross validation procedure needs only to be performed once across the data set and represents a small fraction of the total computational time, the $\mathrm{k}-\mathrm{CV}$ is a more costly operation as the number and size of the calculations is much larger than LOO-CV. The single set cross validation method (Taflanidis et al. 2013) can be used if many quick validations of the metamodel are needed throughout the solution procedure. This method selects one static set to remove from the data set and determine the RMSE between the model response and known removed values over a range of $\theta$. This is equivalent to $\mathrm{k}-\mathrm{CV}$ with one fold and will result in bias in the accuracy of the metamodel response toward those regions of the design space where the error has been assessed, which in turn implies that the $\theta$ predicted is not the true optimum. Loweth et al. (2011) suggested that the k-CV was the best method for obtaining the optimal $\theta$, however it will be shown in Section 4 that LOO-CV is a more effective method here due to the size of the DOE used.

\section{Numerical methods}

\subsection{Design of experiments and metamodel building}

The first stage of the solution procedure was to calculate suitable ranges for the 4-dimensional DOE, the small scale topography parameter $\psi$ has limits between 0 and 1 but the bounds of the small scale input parameters $\frac{d p}{d x}, p, g$ needed to be identified. To do this solutions to the smooth surface EHL problem were calculated using the same solver as described in
Section 3.2 where the MLS metamodels were replaced by the basis functions with all coefficients equal to unity. The maximum/minimum values of $\frac{\mathrm{dp}}{\mathrm{dx}}, \mathrm{p}, \mathrm{g}$ were recorded at convergence and $+/-10 \%$ of the value added/subtracted to give the design space limits of each variable (negative film gaps and pressure are not permitted and were limited to $5 \mu \mathrm{m}$ and $0 \mathrm{~Pa}$ ), the values were rounded and are presented in Table 1.

The DOE was created using the bounds identified and the OLHC permutation GA of Bates et al. (2004). A DOE size of $\mathrm{N}=1000$ was selected as to ensure that the metamodel be accurate over the entire design space, high fidelity results were required over a fast computation because of the sensitivity of the large scale solver to the operating parameters. The justification of this number of points from the resulting metamodel accuracy is presented in Section 4.2.

It is of note that the DOE was built in terms of $\frac{d p}{d x}, p, g, \psi$ so that $\mathrm{q}, \tau, \mathrm{p}^{*}$ were given as functions of these parameters but for the metamodel building and large scale solution $\frac{\mathrm{dp}}{\mathrm{dx}}$ and $\mathrm{q}$ were interchanged so that $\frac{\mathrm{dp}}{\mathrm{dx}}$ is a function of $\mathrm{q}, \mathrm{p}, \mathrm{g}, \psi$. The relations for $\tau$ and $p^{*}$ were not subject to this. In each of the three relations four of the five dimensions were structured such that they meet the space-filling criteria of the OLHC DOE. This ensures that when the MLS metamodels were calculated, the resulting approximation accuracy was not biased toward certain regions of the design space.

Each of the 1000 small scale simulations specified by the DOE were calculated and the outputs recorded. The values for all constants and operating conditions are given in Table 2. COMSOL Multiphysics (COMSOL 2015) was used to solve the range of small scale fluid structure interaction problems as defined in Section 2.2. The MLS metamodels were then calibrated by performing LOO-CV and $\mathrm{k}-\mathrm{CV}$ on each of the three relations, the results produced were analysed and the optimal closeness of fit selected to within an acceptable significance. This was achieved using codes developed with MATLAB (MathWorks 2015) as is also the case for remainder of the solution procedure described.

\subsection{Large scale solution procedure}

The large scale solution procedure began with an initial guess of zero pressure $\mathrm{p}$, the undeformed film thickness $\mathrm{h}$ (specified by a minimum $h_{b}$, pad length $L_{p}$ and tilt angle $\varphi$ ) and an arbitrary guess for the mass flow rate per unit depth $\mathrm{q}$

Table 1 Bounds for the small scale input parameters

\begin{tabular}{lll}
\hline Parameter & Bounds & Unit \\
\hline$\frac{\mathrm{dp}}{\mathrm{dx}}$ & {$[-40,10]$} & $\mathrm{MPa} / \mathrm{mm}$ \\
$\mathrm{p}$ & {$[0,10]$} & $\mathrm{MPa}$ \\
$\mathrm{g}$ & {$[5,50]$} & $\mu \mathrm{m}$ \\
\hline
\end{tabular}


Table 2 Constants and operating conditions

\begin{tabular}{lll}
\hline Parameter & Value/range & Unit \\
\hline $\mathrm{E}$ & 0.5 & $\mathrm{GPa}$ \\
$\mathrm{k}_{1}$ & 0.4667 & $\mu \mathrm{m} / \mathrm{MPa}$ \\
$\mathrm{L}$ & 10 & $\mu \mathrm{m}$ \\
$\mathrm{L}_{\mathrm{p}}$ & 25 & $\mathrm{~mm}$ \\
$\mathrm{U}$ & 1 & $\mathrm{~m} / \mathrm{s}$ \\
$\mathrm{W}$ & 100 & $\mathrm{kN}$ \\
$\alpha$ & 7.5 & $\mu \mathrm{m}$ \\
$\eta_{0}$ & 1 & $\mathrm{~Pa} . \mathrm{s}$ \\
$\nu$ & 0.4 & - \\
$\rho_{0}$ & 870 & $\mathrm{~kg} / \mathrm{m}^{3}$ \\
$\tau_{0}$ & 5 & $\mathrm{~N} / \mathrm{mm}^{2}$ \\
$\varphi$ & 0.05 & $\mathrm{deg}$ \\
\hline
\end{tabular}

(constant along the length of the bearing (7)). The small scale topography parameter $\psi$ was set to be constant along the length of the bearing. This was not a limitation to the method as it is conceivable that topography could vary along the pad length and indeed this variation could be parameterised and optimised in the same approach as described in Section 3.3.

Two different methods were used to satisfy the boundary conditions for pressure (8) by treating the solution of the pressure gradient Eq. (6) either as an Initial Value Problem (IVP) or a Boundary Value Problem (BVP). The IVP method integrated (6) using the MATLAB function ode45 from the known inlet boundary condition of $\mathrm{p}_{\mathrm{a}}=0$, the error in the outlet pressure boundary condition $\mathrm{p}_{\mathrm{b}} \neq 0$ dictated whether $\mathrm{q}$ had been over or under predicted. An iterative procedure was formed where $\mathrm{q}$ is increased if $\mathrm{p}_{\mathrm{b}}>0$ and decreased if $\mathrm{p}_{\mathrm{b}}<0$. The BVP method used the MATLAB function bvp4c to specify $p_{a}=p_{b}=0$ and solves (6) and (7) together to give $p$ and $q$. This required a good initial guess for both variables, initially this was determined by solving the smooth surface pressure gradient equation with the IVP method as described above.

Once $p_{b}=0$ was satisfied to within a tolerance of $10^{-3}$ the large scale film gap $g$ was updated according the deformation matrix operation (10). The load per unit area $\mathrm{p}^{*}$ was assessed and relaxed (given an initial value of zero) with a factor of 0.5 to the previous iteration as to ensure convergence of $g$. After the new $g$ was determined $\mathrm{p}$ and $\mathrm{q}$ were updated using the IVP or BVP methods, with the current values forming the new initial guesses. This continued until convergence in the pressure distribution was achieved, a tolerance of $10^{-3}$ was required. Using the converged solution the shear stress $\tau$ was calculated. The load capacity $\mathrm{W}$ (5) and coefficient of friction $\mu$ (4) were then recorded.

In order to ensure that the load capacity constraint (3) was satisfied a bisector approach was used, which is an appropriate method given that $\mathrm{W}$ reduces monotonically with increasing $h_{b}$. Two values of $h_{b}$ were selected and the corresponding $W$ calculated using the method as described above. A straight line approximation was made from this $\mathrm{W}-\mathrm{h}_{\mathrm{b}}$ relationship which is then used to predict the $h_{b}$ where $W_{\text {req }}$ was achieved. The limits of $h_{b}$ in the straight line approximation were adjusted to include the new value and then the new $\mathrm{W}$ was calculated. This process was repeated until convergence within a tolerance of $10^{-3}$ in $\mathrm{W}$ was achieved.

\subsection{Optimisation of small scale topography}

In order to determine the value of $\psi$ which produced the minimum $\mu$ for the bearing a 1D bracketing optimisation approach was used (Forrester et al. 2008). A parametric assessment of $\mu$ was made over the full range of $\psi$. Any minima of $\mu$ were identified from the response by the locations of where the derivative of $\mu$ with $\psi$ was zero and the second order gradient was positive. These gradients were obtained by finite differences. The assessment ranges were refined around the $\psi$ corresponding to any minimum $\mu$. The parametric assessment was repeated within these new limits and the process was repeated until the minimum value of $\mu$ converged to within a tolerance of $10^{-3}$. After each iteration the ranges were refined by a factor of 0.5 around the new location of the minimum, unless this exceeded either of the initial bounds when that value was used instead.

\section{Results and discussion}

\subsection{Design of experiments and metamodel building}

All results presented were calculated using a 6-core machine with $32 \mathrm{~GB}$ of RAM, running at $3.5 \mathrm{GHz}$. Figure 3 shows the frequency histogram of minimum normalised distance of each point to another point in the DOE. The range of minimum distances shown in Fig. 3 illustrates that the DOE is relatively well conditioned because the frequency distribution is close to normal and the variance is small, indicating that the OLHC has reduced the spread of the minimum distance to other points across all points in the domain. An outlier does exist and there is a slight skew in the distribution toward larger distances between points. The size of the DOE $(\mathrm{N}=1000)$ and the spacefilling criteria of the OLHC ensures that there is enough information for the metamodel approximations to be accurate over the entire design space, as shown in Section 4.2.

The process of running all 1000 small scale simulations took $\sim 11$ days of calculation. Cross validation for the MLS metamodel building was performed, the result of this for the pressure gradient metamodel is given in Fig. 4.

Figure 4 indicates that the optimal closeness of fit for the pressure gradient metamodel obtained from k-CV (with $\mathrm{k}=120$ ) and LOO-CV are very close together, $\mathrm{k}-\mathrm{CV}$ gives $\theta=39.09$ and LOO-CV gives $\theta=40.13$. This shows that both cross validation methods can be used to perform accurate analysis of the closeness of fit response and that the best 


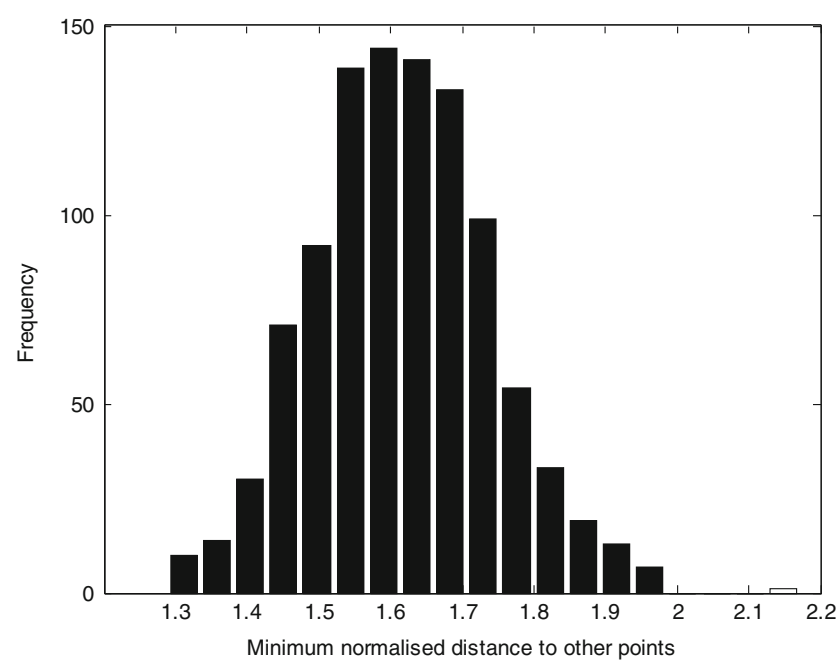

Fig. 3 Frequency histogram of the minimum normalised distance between DOE points

closeness of fit for this DOE data is, to within an acceptable significance, 40. Similar conclusions can be drawn for the load per unit area and shear stress metamodels, in these cases the optimal closeness of fits were found to be 24 and 38 respectively.

Each LOO-CV procedure took less than 5 min to complete whereas the $\mathrm{k}-\mathrm{CV}$ procedures took more than $2 \mathrm{~h}$ to run, this is because the k-CV method requires many more calls to the MLS assessment function than LOO-CV. It is therefore recommended that LOO-CV should be used for cross validation procedures of this type in the future since there is no benefit in accuracy from employing $\mathrm{k}-\mathrm{CV}$. This is different to the conclusions drawn by Loweth et al. (2011) who suggest that k-CV is a more accurate predictor in the context of their metamodels, their problem was however much smaller $(\mathrm{N}=50)$ and therefore no noticeable difference in computational time was observed. It is also shown in

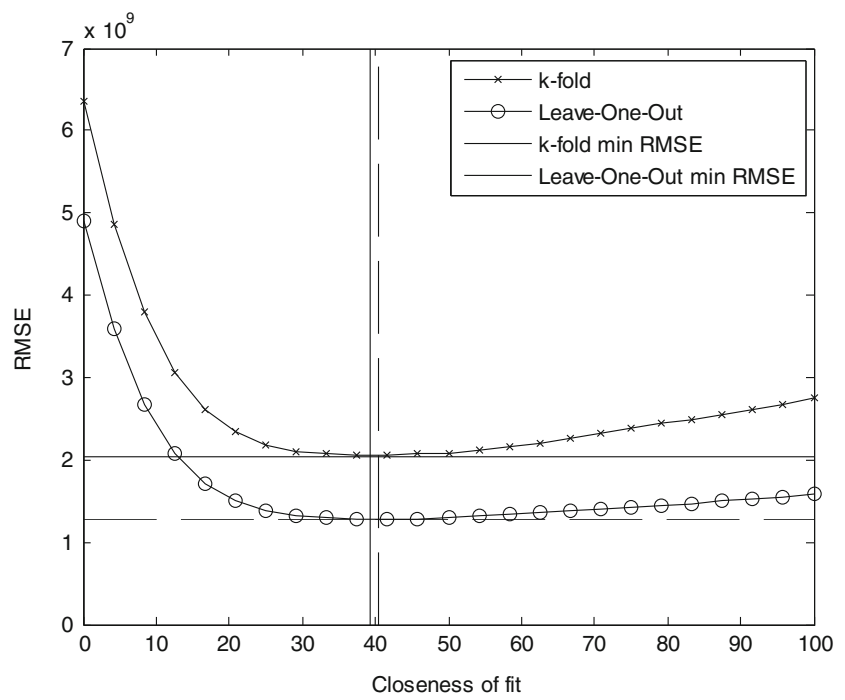

Fig. 4 Cross validation response for the MLS pressure gradient metamodel building

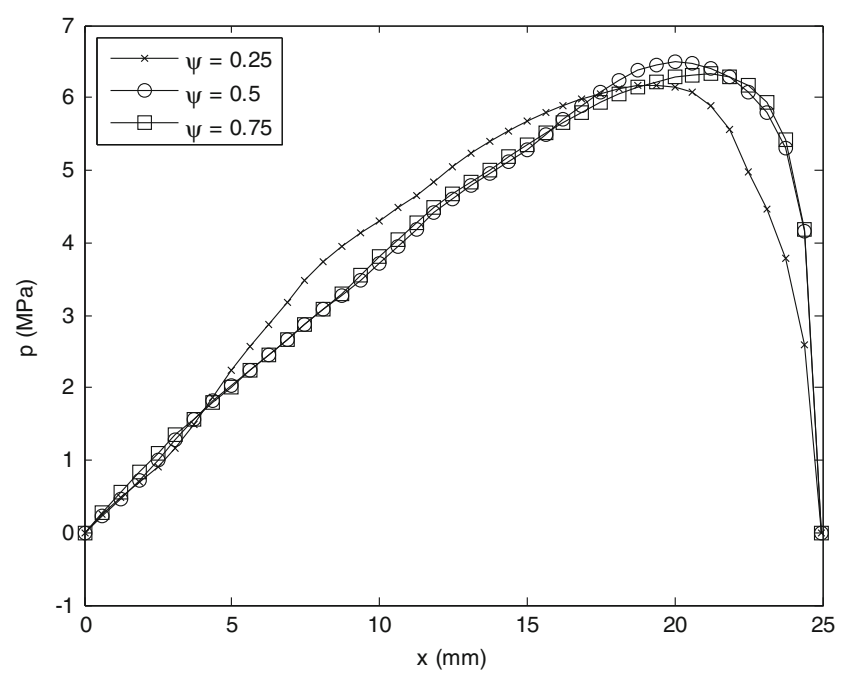

Fig. 5 Pressure distributions for $\psi=0.25,0.5$, and 0.75

Fig. 4 that the LOO-CV error is less than that given by k-CV. This is because the number of DOE points used in LOO-CV to build the validation metamodels is larger than that used in $\mathrm{k}-\mathrm{CV}$ and therefore more in known about the response and a more accurate prediction is made.

\subsection{Two-scale solutions and metamodel accuracy}

Pressure and film gap distributions for three different values of the small scale topography parameter $(\psi=0.25,0.5$, and 0.75$)$ are given in Figs. 5 and 6 respectively. These distributions are generated by solving the large scale problem as described in Section 3.2 (using both IVP and BVP methods) with the MLS metamodels created in Section 4.1.

In order to validate the trends presented in Figs. 5 and 6 results generated at the large scale through the metamodel are compared against the exact corresponding small scale simulations. The mass flow rate as predicted by the large scale solver

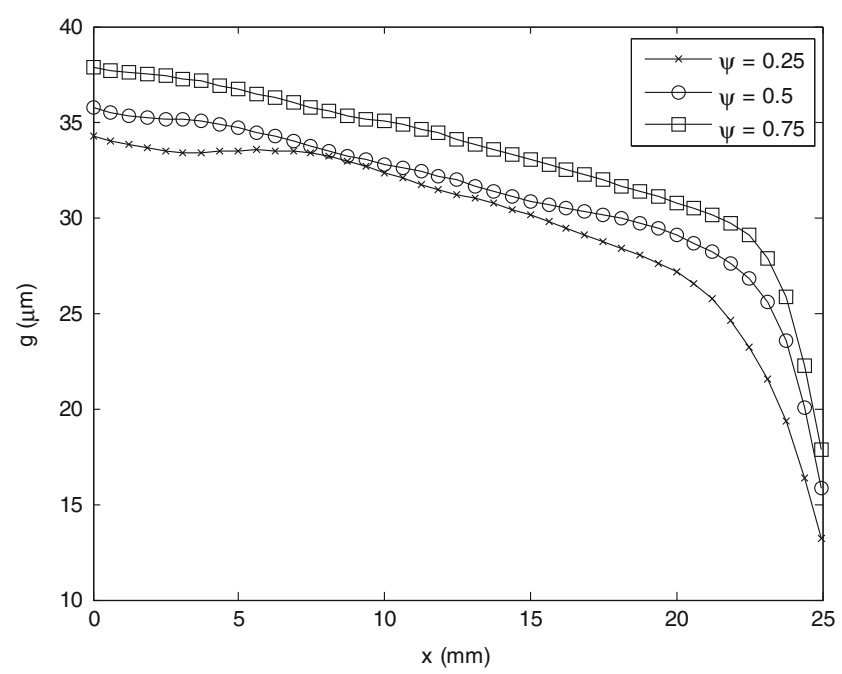

Fig. 6 Film gap distributions for $\psi=0.25,0.5$, and 0.75 
Table 3 Percentage error in mass flow rate for $\psi=0.25$

\begin{tabular}{llll}
\hline Parameter & \multicolumn{2}{l}{ Large scale coordinate } \\
\cline { 2 - 4 } & $0 \mathrm{~mm}$ & $10 \mathrm{~mm}$ & $20 \mathrm{~mm}$ \\
\hline Pressure gradient, $\frac{\mathrm{dp}}{\mathrm{dx}}$ & $0.4302 \mathrm{MPa} / \mathrm{mm}$ & $0.2700 \mathrm{MPa} / \mathrm{mm}$ & $-0.0714 \mathrm{MPa} / \mathrm{mm}$ \\
Pressure, $\mathrm{p}$ & $0 \mathrm{MPa}$ & $4.306 \mathrm{MPa}$ & $6.156 \mathrm{MPa}$ \\
Gap, g & $34.27 \mu \mathrm{m}$ & $32.33 \mu \mathrm{m}$ & $27.11 \mu \mathrm{m}$ \\
$\begin{array}{l}\text { \% error in mass } \\
\text { flow rate }\end{array}$ & $-0.873 \%$ & $3.786 \%$ & $2.213 \%$ \\
\hline
\end{tabular}

is compared to the exact corresponding mass flow rate determined at the small scale for three arbitrary locations $(0,10$, $20 \mathrm{~mm}$ ) along the distributions of pressure gradient, pressure and film gap. This check is performed for each of $\psi=0.25$, 0.5 , and 0.75 with the results tabulated in Tables 3,4 and 5 respectively.

The absolute percentage error in mass flow rate predicted between the metamodel and exact small scale simulations is $<4 \%$ for all cases considered. This indicates that the MLS metamodel is accurately capturing the effects of the parameterised topography on the bearing performance. This also validates the choice in size and spread of the DOE used and implies that the subsequent optimisation procedure will lead to an accurate prediction of the best design. The largest $\%$ error is seen at $\psi=0.25$, here the result predicted is farther from the smooth surface model than for the remaining two cases (see the shape of the distributions given Figs. 10 and 13). This means that the underlying basis function of the metamodel has a poorer fit to the DOE data and the approximation is therefore more likely to be less accurate in the region of the design space as it must deviate further.

Table 6 shows the time to compute for each of the three $\psi$ specified using the IVP and BVP methods for solving the large scale governing equations of flow. The BVP method is shown to be approximately $45 \%$ more efficient than the IVP method across all of the cases investigated, this method was therefore selected as the solution method to be used in the optimisation study. The time saving between the two methods represents a significant improvement from the method derived by de Boer et al. (2014). Table 6 also indicates that at $\psi=0.25$ the solution time for each method was much more than in the

Table 4 Percentage error in mass flow rate for $\psi=0.5$

\begin{tabular}{llll}
\hline Parameter & \multicolumn{2}{l}{ Large scale coordinate } \\
\cline { 2 - 4 } & $0 \mathrm{~mm}$ & $10 \mathrm{~mm}$ & $20 \mathrm{~mm}$ \\
\hline Pressure gradient, $\frac{\mathrm{dp}}{\mathrm{dx}}$ & $0.3658 \mathrm{MPa} / \mathrm{mm}$ & $0.3633 \mathrm{MPa} / \mathrm{mm}$ & $0.0221 \mathrm{MPa} / \mathrm{mm}$ \\
Pressure, $\mathrm{p}$ & $0 \mathrm{MPa}$ & $3.704 \mathrm{MPa}$ & $6.491 \mathrm{MPa}$ \\
$\begin{array}{l}\text { Gap, g } \\
\text { \% error in } \\
\text { mass flow rate }\end{array}$ & $35.71 \mu \mathrm{m}$ & $32.77 \mu \mathrm{m}$ & $29.06 \mu \mathrm{m}$ \\
& $1.198 \%$ & $1.997 \%$ & $-0.225 \%$ \\
\hline
\end{tabular}

Table 5 Percentage error in mass flow rate for $\psi=0.75$

\begin{tabular}{llll}
\hline Parameter & \multicolumn{2}{l}{ Large scale coordinate } \\
\cline { 2 - 4 } & $0 \mathrm{~mm}$ & $10 \mathrm{~mm}$ & $20 \mathrm{~mm}$ \\
\hline Pressure gradient, $\frac{\mathrm{dp}}{\mathrm{dx}}$ & $0.4867 \mathrm{MPa} / \mathrm{mm}$ & $0.4081 \mathrm{MPa} / \mathrm{mm}$ & $0.0777 \mathrm{MPa} / \mathrm{mm}$ \\
Pressure, $\mathrm{p}$ & $0 \mathrm{MPa}$ & $3.798 \mathrm{MPa}$ & $6.282 \mathrm{MPa}$ \\
$\begin{array}{l}\text { Gap, g } \\
\begin{array}{l}\text { \% error in } \\
\text { mass flow rate }\end{array}\end{array}$ & $37.83 \mu \mathrm{m}$ & $35.00 \mu \mathrm{m}$ & $30.76 \mu \mathrm{m}$ \\
\hline
\end{tabular}

other cases, this relates to the accuracy of the metamodel in this region of the design space and the influence of topography causing much larger deviations from the underlying functions of the MLS metamodel.

\subsection{Optimisation of topography}

Using the MLS metamodels validated in Section 4.2, optimisation of topography was performed by the bracketing procedure outlined in Section 3.3. The response and optimisation of $\mu$ with $\psi$ is presented in Fig. 7 , the minimum film gap $g_{b}$ and $q$ corresponding to this are given in Figs. 8 and 9 (not all assessed points are displayed for the purpose of visualisation).

The response shown in Fig. 7 indicates that a transverse topography produces a lower $\mu$ at constant $\mathrm{W}$ than purely longitudinal topography for the conditions investigated. This observation is consistent with that observed by Patir and Cheng (1978) when they conducted computer experiments using their flow factors approach to include the effects of topography in an EHL simulation. The response of $\mu$ with $\psi$ is non-linear, as $\psi$ increases from 0 to 0.65 there is a decrease in $\mu$ from 0.023 to 0.08 , between $\psi=0.65$ and $\psi=0.85 \mu$ remains between a value of 0.08 and 0.09 , and as $\psi$ increases from 0.85 to $1, \mu$ increases from 0.08 to 0.095 . The optimisation procedure took a total of $\sim 15 \mathrm{~h}$ to converge, this accounted for 60 separate assessments of $\mu$ over the specified values of $\psi$.

Two local minima were identified by the optimisation procedure at $\psi=0.6579$ and $\psi=0.8421$ for which $\mu=8.104 \times 10^{-3}$ and $\mu=8.028 \times 10^{-3}$ respectively. The minimum at $\psi=0.8421$ is therefore identified as the global minimum of $\mu$ for the conditions imposed and is therefore the optimal $\psi$ for the bearing design. Figures 8 and 9 show that

Table 6 Time to compute using the IVP and BVP methods

\begin{tabular}{lll}
\hline $\begin{array}{l}\text { Small scale topography } \\
\text { parameter }\end{array}$ & $\begin{array}{l}\text { Time to compute } \\
\text { IVP (s) }\end{array}$ & $\begin{array}{l}\text { Time to compute } \\
\text { BVP (s) }\end{array}$ \\
\hline$\psi=0.25$ & 2219 & 1330 \\
$\psi=0.5$ & 1032 & 573 \\
$\psi=0.75$ & 1112 & 697 \\
\hline
\end{tabular}




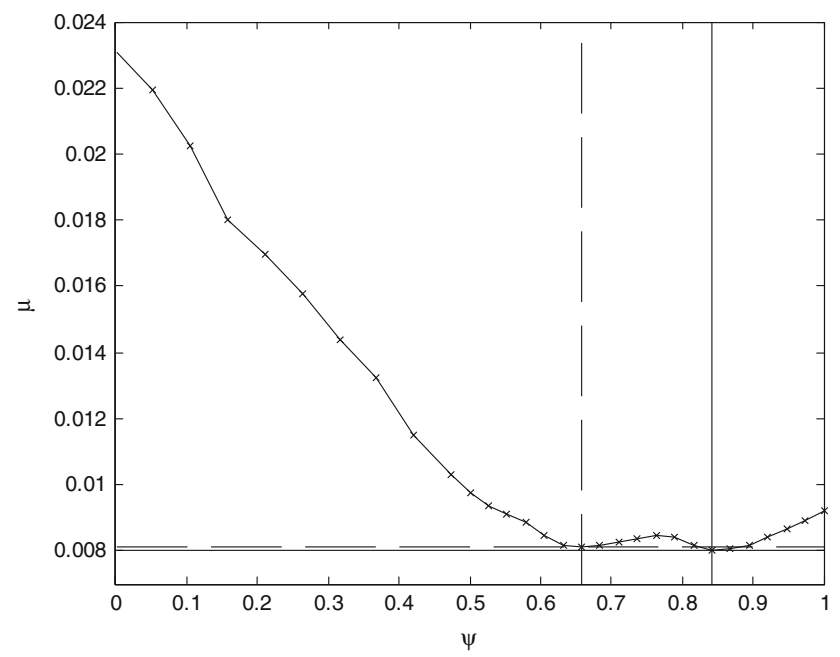

Fig. 7 Response The coefficient of friction as a function of the small scale topography parameter

a decrease in $\mu$ leads to an increase $g_{b}$ and $q$ and an increase in $\mu$ leads to an decrease $g_{b}$ and $q$, such that the minima identified for $\mu$ correspond to maxima of $g_{b}$ and q. Figures 10 and 11 illustrate the topography at the two minima identified.

Given that the coefficients of friction calculated at $\psi=0.6579$ and $\psi=0.8421$ are similar it is interesting to note that they have very different features. In Fig. 11 the topography is dominated by transverse waviness, whereas in Fig. 10 the topography is much more of a blend between transverse and longitudinal components. This highlights that the influence of topography on friction is complex and that to describe and optimise the response of $\mu$ with $\psi$ the two-scale method and subsequent metamodels are needed.

The coefficient of friction produced by the smooth surface model with the same parameters was found to be $\mu=7.175 \times 10^{-3}$. Simulations inclusive of topography predicted higher $\mu$ than the smooth surface equivalent. $g_{b}$ and $q$

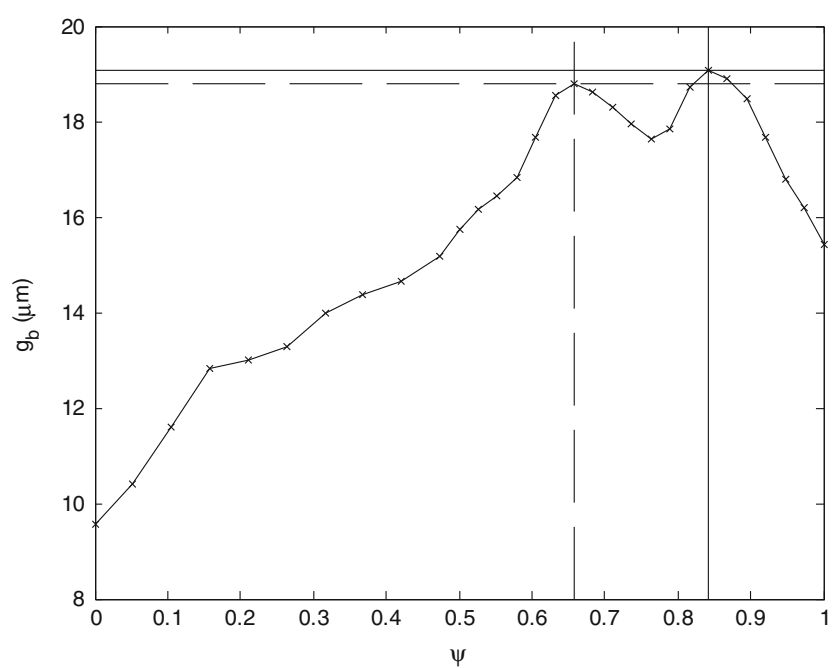

Fig. 8 The minimum film gap as a function of the small scale topography parameter

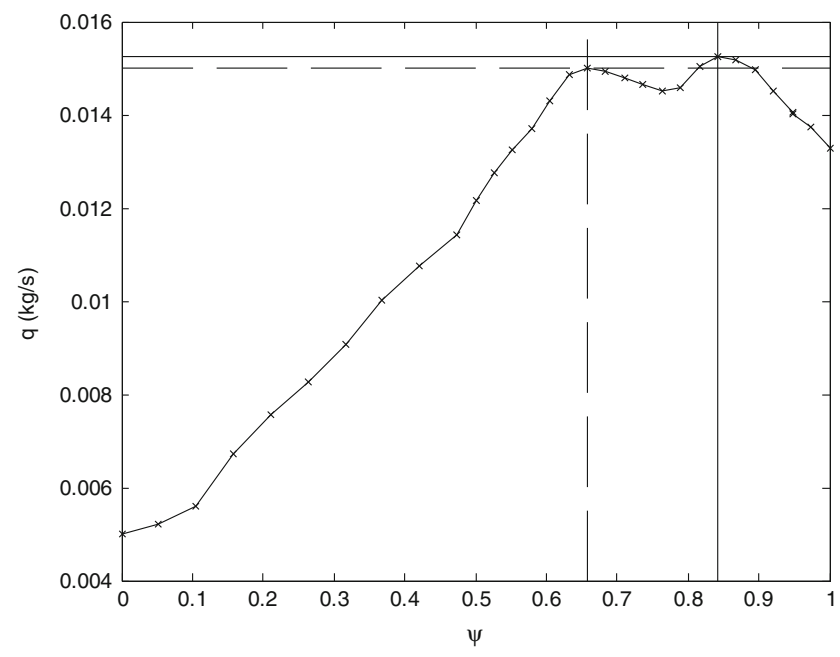

Fig. 9 The mass flow rate per unit depth as a function of the small scale topography parameter

determined using the smooth surface model were found to be $g_{b}=20.15 \mu \mathrm{m}$ and $\mathrm{q}=0.01556 \mathrm{~kg} / \mathrm{s}$ which are both higher than any value determined with topography. These results show the potential of the multiscale approach for analysing the differences in bearing performance when topography is and is not considered. This subsequently demonstrates that when real surfaces are present the orientation of topography as manufactured can be guided by the optimisation process described. This method also provides a comprehensive framework for analysing and optimising surface topography in more complex cases which have been shown to have a role in reducing friction when compared to the smooth surface model, such as cavitating (Gao et al. 2015) and transient (Etsion 2005) lubrication.

The $\mathrm{p}, \frac{\mathrm{dp}}{\mathrm{dx}}, \mathrm{p}^{*}, \mathrm{~g}$, and $\tau$ distributions for the textured bearing under load are shown in Figs. 12, 13, 14, 15 and 16 at the two minima identified in Fig. 7 and directly from the smooth

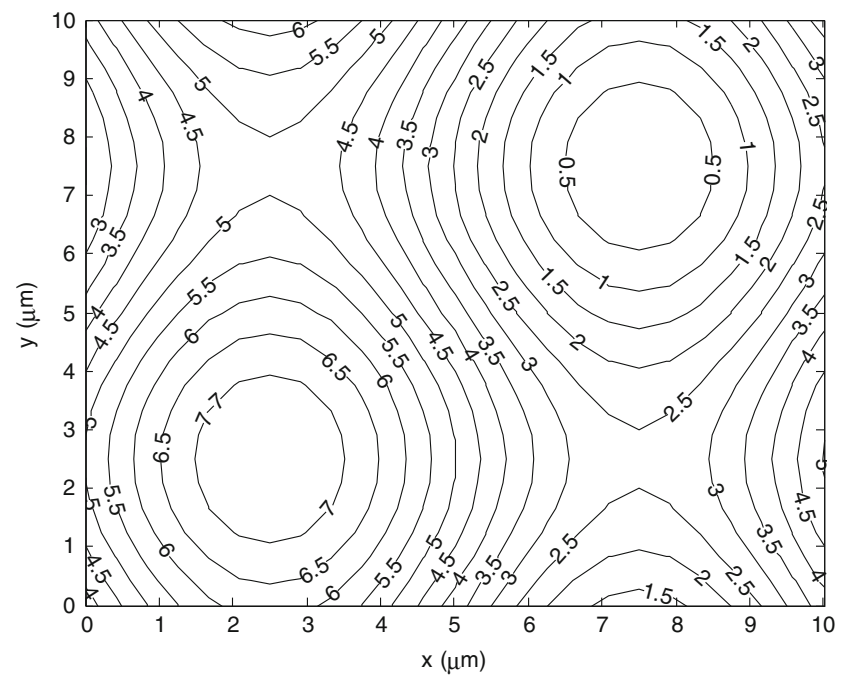

Fig. 10 Contour plot of topography in $\mu \mathrm{m}$ at $\psi=0.6579$ (local minimum) 


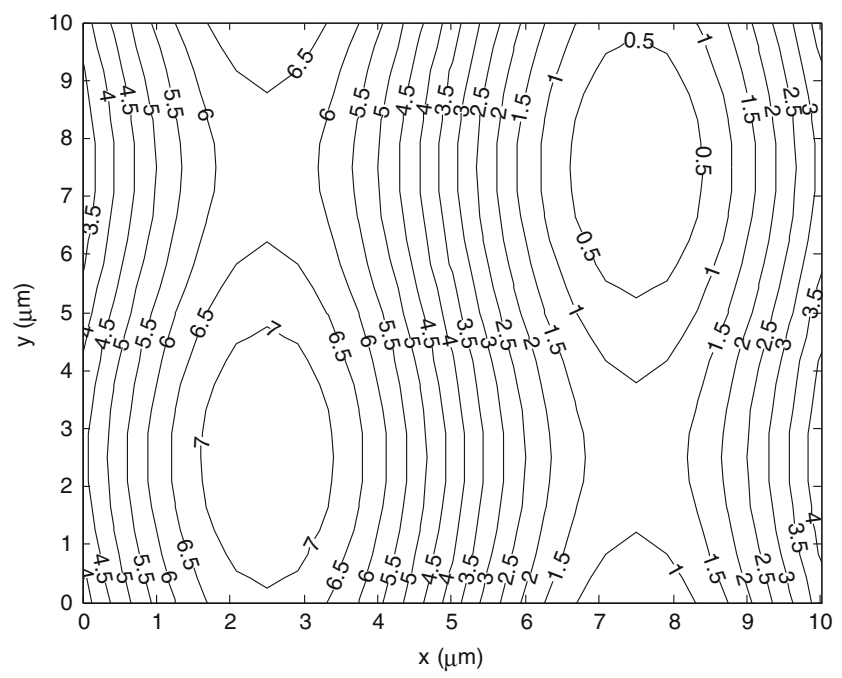

Fig. 11 Contour plot of topography in $\mu \mathrm{m}$ at $\psi=0.8421$ (global minimum)

surface model (without topography or fluid flow phenomena) respectively.

Figure 12 shows that the pressure distributions obtained from the smooth surface model are similar in both shape and magnitude to that obtained with topography. Because the load capacity of each distribution is equal the differences in performance identified with topography cause only a small deviation from the smooth surface model in terms of pressure. This also implies that deviations in the pressure gradient will also be small and this is confirmed in Fig. 13, these changes are small but the influence they have on the remaining part of the calculation is significant and the solution for each distribution is not trivial. The load per unit area shown in Fig. 14 indicates that this is almost identical to the pressure, where the difference between these two distributions is orders of magnitude smaller than the pressure. The pressure distributions show that for the two $\mu$ identified $\psi=0.8421$ produces a higher pressure

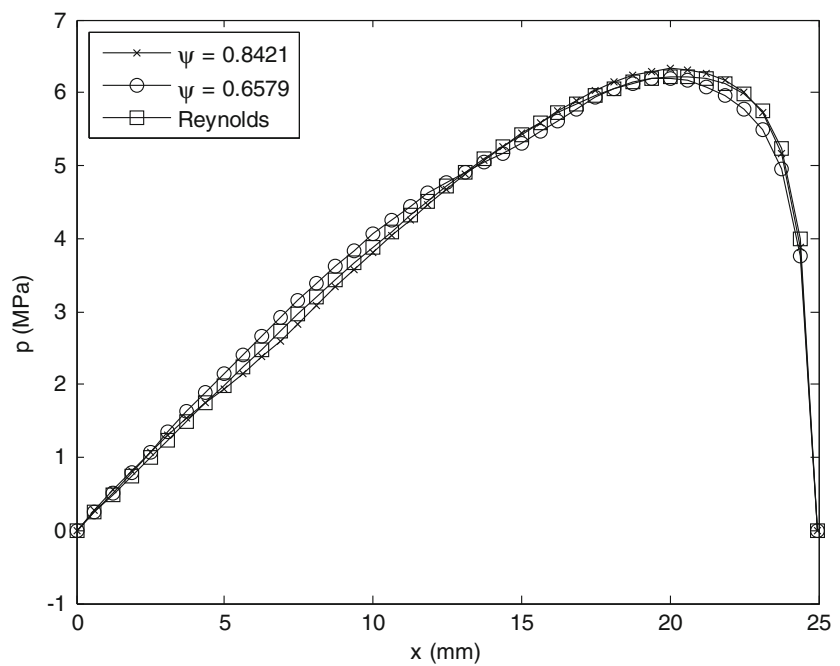

Fig. 12 Pressure distributions for $\psi=0.6579,0.8421$ and the smooth surface model

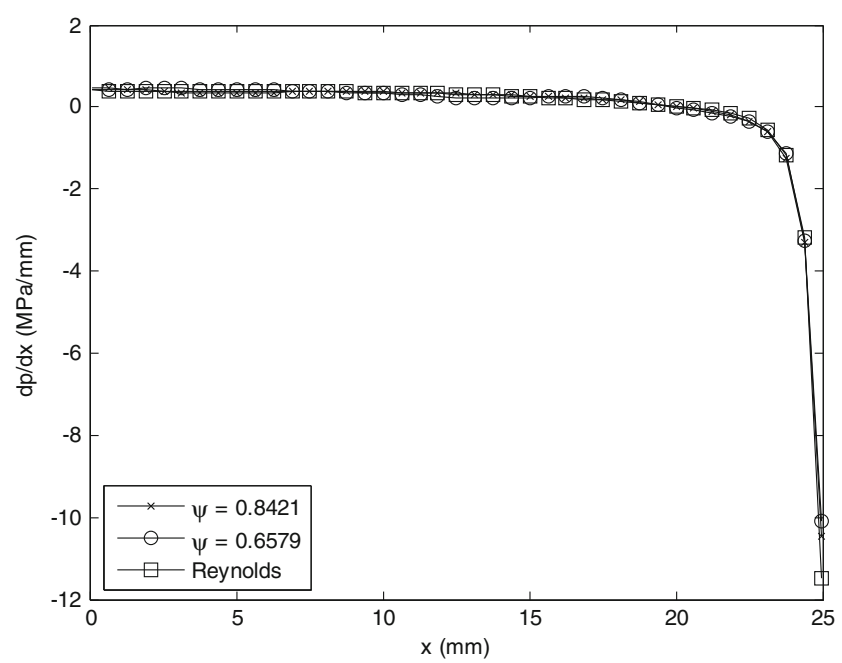

Fig. 13 Pressure gradient distributions for $\psi=0.6579,0.8421$ and the smooth surface model

which occurs further toward the outlet of the bearing than for $\psi=0.6579$ and the pressure at $\psi=0.8421$ is lower than $\psi=0.6579$ over most of the length of the bearing. This is an interesting observation because it indicates the difference in pressure distributions which result in similar coefficients of friction.

The film gap distributions presented in Fig. 15 show that a lower $\mathrm{g}$ is predicted for bearings with topography over the length of the bearing when compared to the smooth surface case. It is also observed that for the two topographies shown the shapes of the film gap distributions are more comparable to each other than they are with the smooth surface model. Implying that with topography similar values of $\mu$ are given when the shape of the film gaps are also similar.

The film gap distributions presented in Fig. 15 show that with topography a lower $g$ is predicted over the length of the

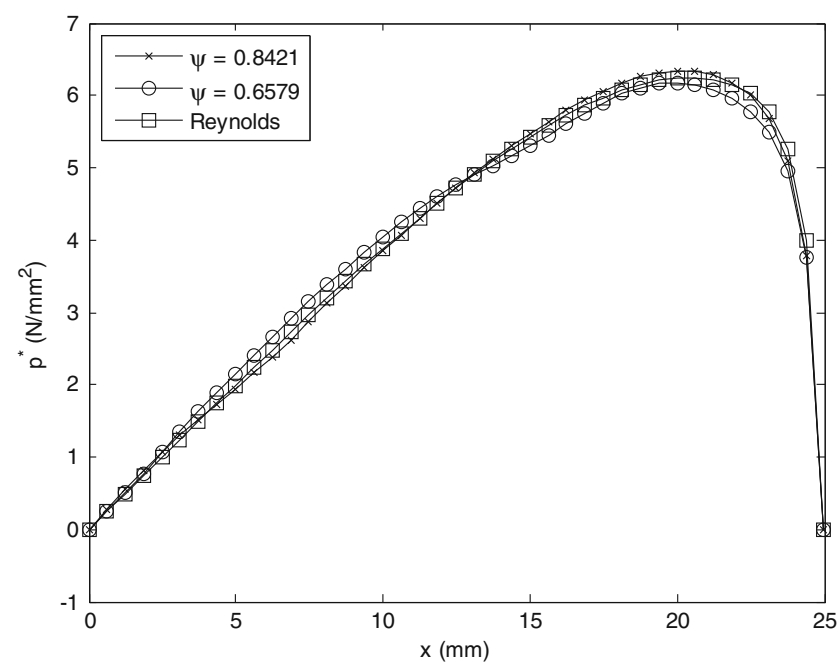

Fig. 14 Load per unit area distributions for $\psi=0.6579,0.8421$ and the smooth surface model 


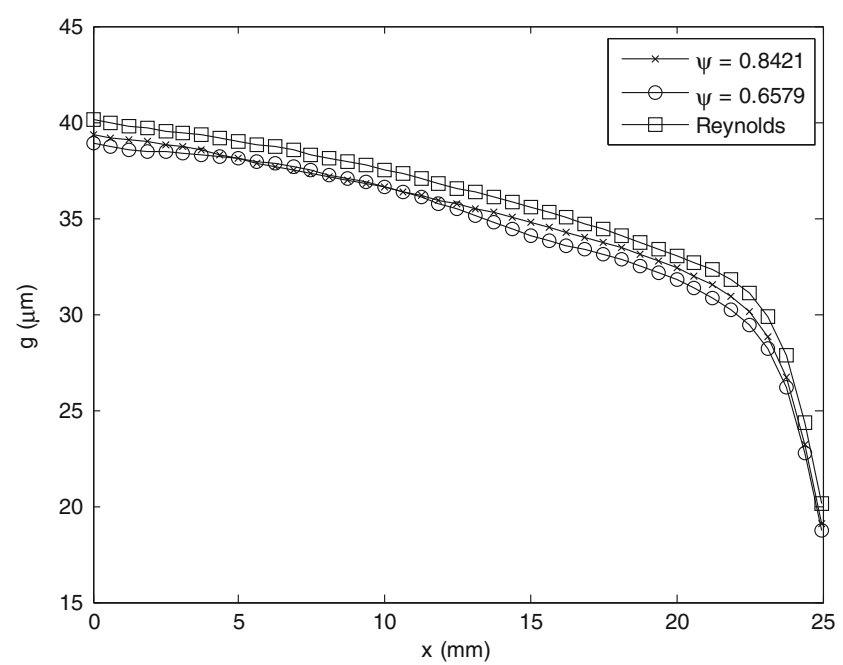

Fig. 15 Film thickness distributions for $\psi=0.6579,0.8421$ and the smooth surface model

bearing when compared to the smooth surface case. It is also observed that for the two topographies shown the shapes of the film gap distributions are more comparable to each other than they are with the smooth surface model. Implying that with topography similar values of $\mu$ are given when the shape of the film gaps are also similar. The film gap distribution given at $\psi=0.8421$ is lower than $\psi=0.6579$ which indicates that under these conditions the minimum $\mu$ for a bearing with topography is synonymous with a minimum for the film gap over the length of the bearing.

The shear stress distributions in Fig. 16 show that for bearings with topography included a larger value is predicted over the length of the bearing than in the smooth surface case, leading to large values of $\mu$. Both distributions with topography exhibit a similar shape and magnitude which explains why the $\mu$ predicted at these $\psi$ are also similar.

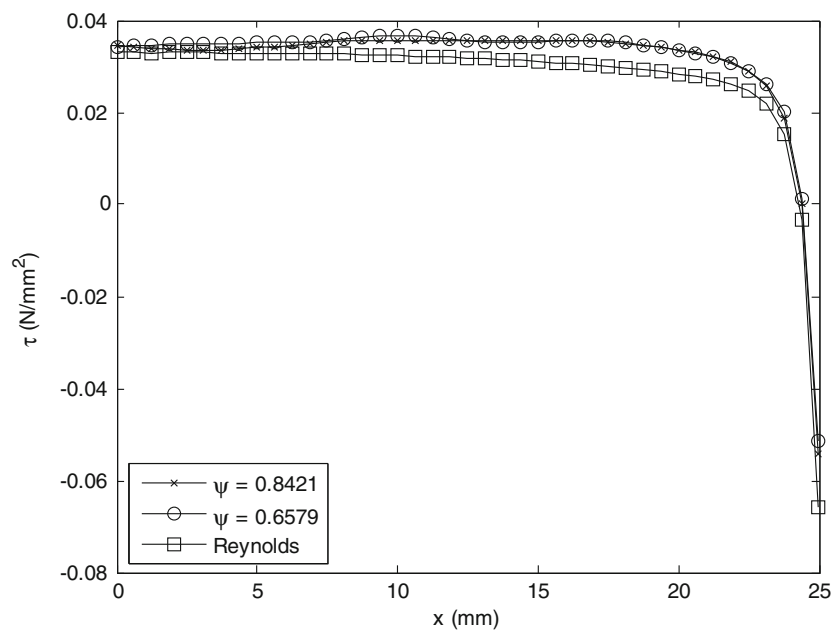

Fig. 16 Shear stress distributions for $\psi=0.6579,0.8421$ and the smooth surface model

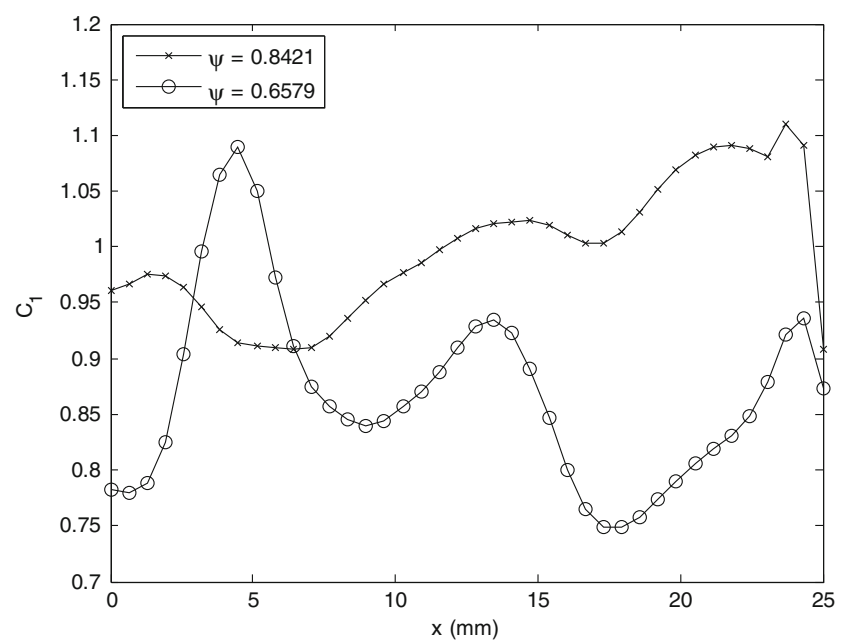

Fig. 17 MLS coefficient $C_{1}$ distributions for $\psi=0.6579$ and 0.8421

The MLS coefficients generated from the pressure gradient metamodel $\mathrm{C}_{1-3}$ are plotted over the length of the bearing for the two minima identified in Figs. 17, 18 and 19.

Each coefficient distribution represents how far from the smooth surface approximation (with $\mathrm{C}_{1-3}=1$ ) the metamodel deviates due to the influence of topography as determined by the small scale simulations. Figures 17, 18 and 19 show that topography introduces a non-linear response for each coefficient since there are no obviously identifiable trends in the responses over the length of the bearing. The coefficients produced at $\psi=0.6579$ and $\psi=0.8421$ indicate that very different characteristics are introduced by the topographies and that in order to accurately model these effects the metamodel approach is required. Also because the coefficient of friction produced by at $\psi=0.6579$ and $\psi=0.8421$ are close together and the MLS coefficients have no clear trends between them, this further implies that in order to conduct an optimisation study over a range of topographies that the two-scale method and subsequent metamodelling techniques are required.

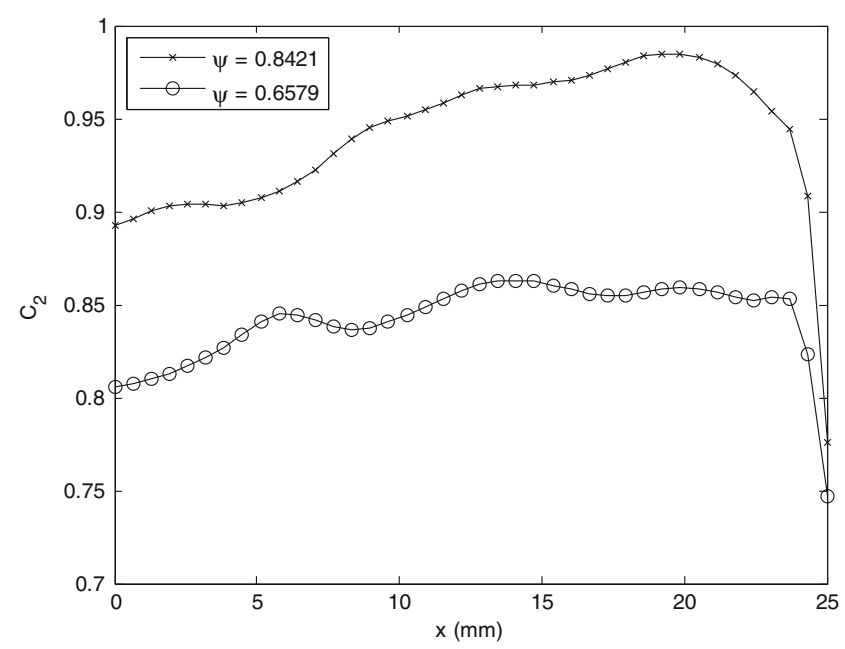

Fig. 18 MLS coefficient $C_{2}$ distributions for $\psi=0.6579$ and 0.8421 


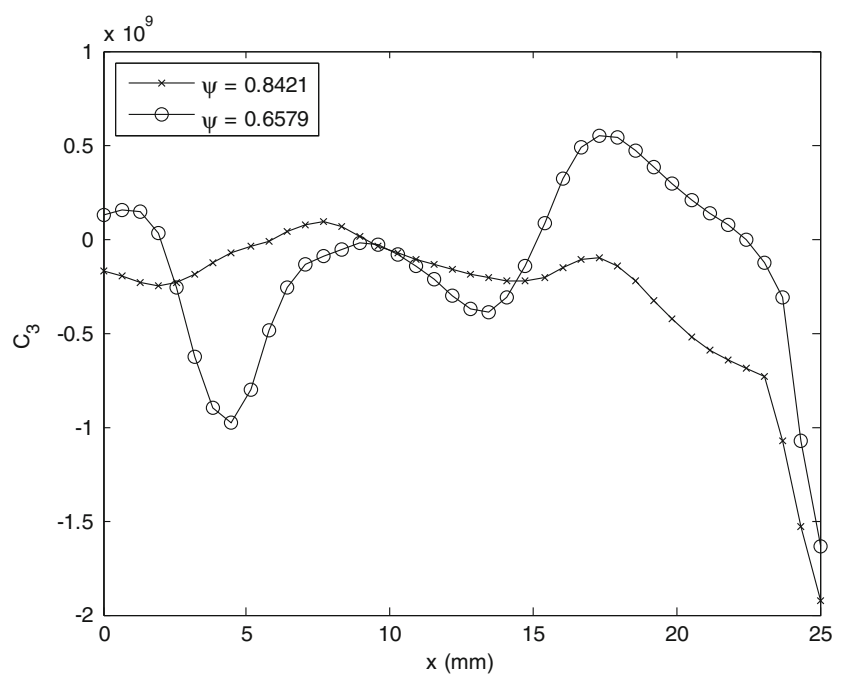

Fig. 19 MLS coefficient $C_{3}$ distributions for $\psi=0.6579$ and 0.8421

\section{Conclusion}

Surface topography influences the friction in lubricated surfaces under load. Developing theoretical methods to optimise topography to, for example, minimise friction is complicated by the separation in scales between the size of the lubricated domain and the topography, the latter being at least an order of magnitude smaller. The separation in scales means that it is infeasible to computationally resolve the small scale features as well as the large scale bearing domain within a single computation.

The present study has shown how metamodels can be used within an efficient two-scale method to reduce friction in EHL bearings by optimising the small scale topography. Accurate metamodels are needed to represent the small scale data and it has been shown that combining OHLC DOE techniques with MLS metamodels that this can provide the accuracy needed. It is also found that there is very little difference between calibration of the MLS closeness of fit parameter using either the LOO-CV or k-CV methods and, given the significantly better computational efficiency of the former, LOO-CV is recommended for the calibration of the EHL metamodels. Note that this preference for LOO-CV over k-CV contrasts with the recent finding of Loweth et al. (2011) for much smaller sized data than is being used here. Another key finding is that the BVP method for determining pressure is over $40 \%$ more efficient than the IVP method and offers a significant improvement over the two-scale approach developed recently by de Boer et al. (2014). It is found that the MLS coefficients are strongly and non-linearly dependent on the topography and the EHL results show that very different topographies can lead to similar friction coefficients. Results also show further that, under a fixed load, transverse topography produces a lower friction coefficient than longitudinal topography, as is consistent with Patir and Cheng (1978), and this demonstrates that in order to optimise surface topography the two-scale method is required.

Acknowledgments The authors would like to thank the Engineering and Physical Sciences Research Council (Grant number EP/1013733/1) and the Leverhulme Trust (Grant Number F10 100/B) for funding this work.

Open Access This article is distributed under the terms of the Creative Commons Attribution 4.0 International License (http:// creativecommons.org/licenses/by/4.0/), which permits unrestricted use, distribution, and reproduction in any medium, provided you give appropriate credit to the original author(s) and the source, provide a link to the Creative Commons license, and indicate if changes were made.

\section{References}

Abraham FF, Broughton JQ, Bernstein N, Kaxiras E (1999) Concurrent coupling of length scales: methodology and application. Phys Rev B 60(4):2391-2402

Audze P, Eglais V (1977) New approach for planning out of experiments, problems of dynamics and strengths, vol 35. Zinatne Publishing House, Riga, pp 104-107

Bates SJ, Sienz J, Toropov VV (2004) Formulation of the optimal latin hypercube designs of experiments. In Proceedings of the 45th AIAA/ ASME/ ASCE/ AHS/ ASC Structures, Structural Dynamics \& Materials Conference. Palm Springs, USA

Benke M, Shapiro E, Drikakis D (2009) Modelling the polymer migration phenomena in DNA-laden flows. 2nd Micro and Nano Flows Conference, West London, UK

Breitkopf P, Naceur H, Rassineux A, Villon P (2005) Moving least squares response surface approximation: formulation and metal forming applications. Comput Struct 83:17-18

Cameron A (1971) Basic lubrication theory. Longman, London

Choi KK, Youn B, Yang R-J (2001) Moving least squares method for reliability-based design optimization, 4th World Congress of Structural and Multidiscpilinary Optimization, Dalian, China

COMSOL (2015). User guide. [pdf] COMSOL. Avaliable at: (http://cdn. comsol.com/documentation/5.1.0.234/COMSOL_ServerManual. pdf) [Accessed 1st October 2015]

de Boer GN, Hewson RW, Thompson HM, Gao L, Toropov VV (2014) Two-scale EHL: three-dimensional topography in tilted-pad bearings. Tribol Int 79:111-125

de Kraker A, van Ostayen RAJ, van Beek A, Rixen DJ (2007) A multiscale method modelling surface texture effects. J Tribol 129: $221-230$

Dowson D (1999) History of tribology. Wiley, New York

Dowson D, Higginson GR (1966) Elastohydrodynamic lubrication: the fundamentals of roller gear lubrication. Pergamon, Oxford

E W, Engquist B, Li X, Ren W, Vanden-Eijnden E (2007) Heterogeneous multiscale methods: a review. Commun Comput Phys 2(3):367-450

Etsion I (2005) State of the art in laser surface texturing. J Tribol 127(1): 248-253

Forrester A, Sobester A, Keane AJ (2008) Engineering design via surrogate modelling: a practical guide. Wiley, New York

Gao L, Hewson RW (2012) A multiscale framework for EHL and microEHL. Tribol Trans 55:713-722

Gao L, de Boer GN, Hewson RW (2015) The role of micro-cavitation on EHL: a multiscale mass conserving approach. Tribol Int 90:324-331

Gohar R (2001) Elastohydrodynamics, 2nd edn. Imperial College Press, World Scientific, London 
Hewson RW, Kapur N, Gaskell PH (2011) A two-scale model for discrete cell gravure roll coating. Chem Eng Sci 66(16):3666-3674

Johnson KL, Tevaarwerk JL (1977) The shear thinning behaviour of elastohydrodynamic oil films. Proc R Soc Lond A 356:215-236

Levin D (1998) The approximation power of moving least squares. Math Comput 67:1517-1531

Loweth EL, de Boer GN, Toropov VV (2011) Practical recommendations on the use of moving least squares metamodel building. In Proceedings of the Thirteenth International Conference on Civil, Structural and Environmental Engineering, Chania, Crete, Greece, 2011, Civil-Comp Press

MathWorks (2015) User guide. [pdf] MATLAB. Avaliable at: (https:// www.mathworks.com/help/pdf_doc/matlab/matlab_prog.pdf) [Accessed 1st October 2015]

Patir N, Cheng HS (1978) Average flow model for determining effects of 3dimensional roughness on partial hydrodynamic lubrication. J Lubr Technol Trans ASME 100(1):12-17

Rodkiewicz CM, Yang P (1995) Proposed Tehl solution system for the thrustbearings inclusive of surface deformations. Tribol Trans 38(1):75-85

Roelands C (1966) Correlational aspects of the viscosity-temperaturepressure relationships of lubricating oils, $\mathrm{PhD}$ thesis, Delft University of Technology, Delft, the Netherlands
Roux WJ, Stander N, Haftka RT (1998) Response surface approximations for structural optimization. Int J Numer Methods Eng 42(3): $517-534$

Sahlin F, Larsson R, Almqvist A, Lugt PM, Marklund P (2010) A mixed lubrication model incorporating measured surface topography. Part 1: theory of flow factors. Proc Inst Mech Eng J Eng Tribol 224(4): 335-351

Taflanidis AA, Jia G, Kennedy AB, Smith JM (2013) Implementation/ optimization of moving least squares response surfaces for approximation of hurricane/storm surge and wave responses. Nat Hazards 66:955-983

Toropov VV, Schramm U, Sahai A, Jones RD, Zeguer T (2005) Design optimization and stochastic analysis based on the moving least squares method, 6th World Congress of Strcutural and Multidiscpilinary Optimization, Rio de Janerio, Brazil

Tzeng ST, Saibel E (1967) Surface roughness effect on slider bearing lubrication. ASLE Trans 10(3):334-348

Writz D, Karajan N, Haasdonk B (2015) Surrogate modeling of multiscale models using kernel methods. Int J Numer Methods Eng 101(1):1-28 